\title{
Probing Bilayer Grain Boundaries in Large-Area Graphene with Tip-Enhanced Raman Spectroscopy
}

\author{
Kyoung-Duck Park, Markus B. Raschke, Joanna M. Atkin, Young Hee Lee, \\ and Mun Seok Jeong*
}

Large-area graphene sheets grown by chemical vapor deposition (CVD) have been proposed as a route to realize mass production and commercialization of near-ballistic transport electronic devices..$^{[1,2]}$ So far, most large-area synthetic graphene is polycrystalline. The associated grain boundaries $(\mathrm{GBs})^{[1,3-5]}$ have been identified as a major limitation in device applications due to their modified electronic structure and inhibiting carrier transport. ${ }^{[6,7]}$ Therefore, understanding the detailed characteristics of GBs is a prerequisite for better design of graphene sheets.

Despite the greater applicability of large-area graphene sheets compared to graphene flakes, most theoretical and experimental studies of GBs have focused on the analysis of atomic defects observed in adjacent crystal faces, ${ }^{[8]}$ which show significant differences from larger scale deformations of GBs in large-area graphene. Scanning tunneling microscopy (STM) and transmission electron microscopy (TEM) can investigate structural defects, electrical conductivity, and strain associated with GBs with atomic scale spatial resolution..$^{[4,9-11]}$ However, these high-resolution local probes provide only a limited field of view, without optical and chemical information. In contrast, Raman spectroscopy and imaging studies of GBs in large-area graphene have shown both an increase and decrease in the $G^{\prime}$ peak intensity at GBs, ${ }^{[1,5,12]}$ and thus have left a confusing picture regarding their lattice structure and vibrational properties, in part due to the diffraction-limited spatial resolution and a low sensitivity ${ }^{[8]}$ of conventional Raman spectroscopy.

In order to complement STM, TEM, and conventional Raman spectroscopy and to bridge their length scales, here we present $\approx 18 \mathrm{~nm}$ spatial resolution tip-enhanced Raman

\author{
K.-D. Park, Prof. M. B. Raschke \\ Department of Physics \\ Department of Chemistry, and JILA \\ University of Colorado \\ Boulder, CO 80309, USA \\ Prof. J. M. Atkin \\ Department of Chemistry \\ University of North Carolina \\ Chapel Hill, NC 27599, USA \\ Prof. Y. H. Lee, Prof. M. S. Jeong \\ Center for Integrated Nanostructure Physics \\ Institute for Basic Science \\ Sungkyunkwan University \\ Suwon 440-746, Republic of Korea \\ E-mail:mjeong@skku.edu \\ Prof. Y. H. Lee, Prof. M. S. Jeong \\ Department of Energy Science \\ Sungkyunkwan University \\ Suwon 440-746, Republic of Korea
}

DOI: 10.1002/adma.201603601 spectroscopy (TERS) and imaging to understand lattice and electronic structure, as well as elastic and phonon scattering properties of GBs in CVD grown large-area graphene. Through correlated analysis of topography, near-field scattering, and multispectral TERS imaging, we reveal bilayer GBs in the form of twisted stacking at the boundary between two misoriented crystal facet. We also determine the misorientation angles of the bilayer GBs from a detailed quantitative investigation of the Raman $G$ and $G^{\prime}$ peak properties, which varying with respect to the misorientation angle associated with the modified electronic structure at the K-point of the Brillouin zone.

Further, we investigate the distinct Raman characteristics from a wrinkle, a different type of line defect. In contrast to the GBs, only the $G^{\prime}$ peak exhibits significant changes in intensity with no associated spectral shift in the vicinity of the wrinkle, which directly correlated with a nanoscopic structural curvature effect. From multispectral TERS mapping, we also resolve the chemical variation associated with nucleation sites (NSs), where we confirm AB stacking bilayer structures at the NS and find an unexpected phonon scattering response of the $\mathrm{D}^{\prime}$ peak, which is associated with $\mathrm{sp}^{3}$-type carbon hybridization defects. Beyond the newly revealed properties of the defects, these results may provide clues to understand the growth mechanism of large-area graphene and the formation of nanoscale defects. Furthermore, our work demonstrates the potential of correlated analysis of multispectral TERS imaging as a generally applicable approach for the investigation of the wide range of $2 \mathrm{D}$ materials beyond graphene.

We studied large-area graphene, grown on copper foil using $\mathrm{CVD}^{[13]}$ and transferred onto a glass cover slip. The multispectral TERS imaging system used in these experiments was based on a modified commercial confocal Raman imaging system (NTEGRA spectra, NT-MDT), as shown in Figure 1a,b. A helium-neon laser ( $632.8 \mathrm{~nm}, P<2 \mathrm{~mW}$ ) was focused onto the transferred monolayer (ML) graphene sample by an oil immersion objective lens (NA = 1.3), with the linear polarization effectively exciting in-plane vibrational modes (Raman D, $G$, and $G^{\prime}$ peaks) as described in Figure 1b,c. The Au tip of the shear-force atomic force microscopy (AFM) was positioned in the focal spot to enhance the Raman scattering signal by localized surface plasmon resonance (LSPR) and to increase the spatial resolution for nanoscale TERS imaging. The near-field Rayleigh scattering signal from the $\mathrm{Au}$ tip was simultaneously measured, providing an approximate measure of the absorption of the thin graphene films. Confocal Raman measurements were performed with the Au tip retracted from sample.

For precharacterization, topography and near-field Rayleigh scattering images of a wrinkle (indicated by W) and grain boundary (indicated by GB) structure on transferred 


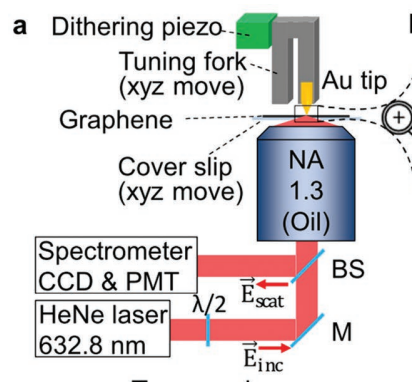

a Dithering piezo. Tuning fork
(xyz move)

Graphene

Cover slip (xyz move) Topography

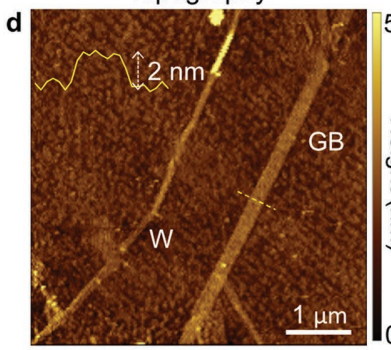

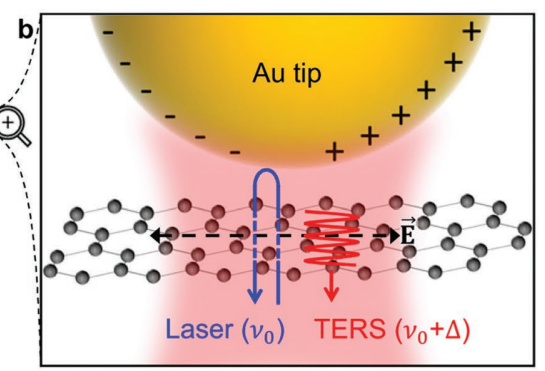

c In-Plane Phonon Vibrations

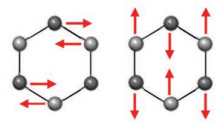

$\mathrm{G}$ peak at $\Gamma$
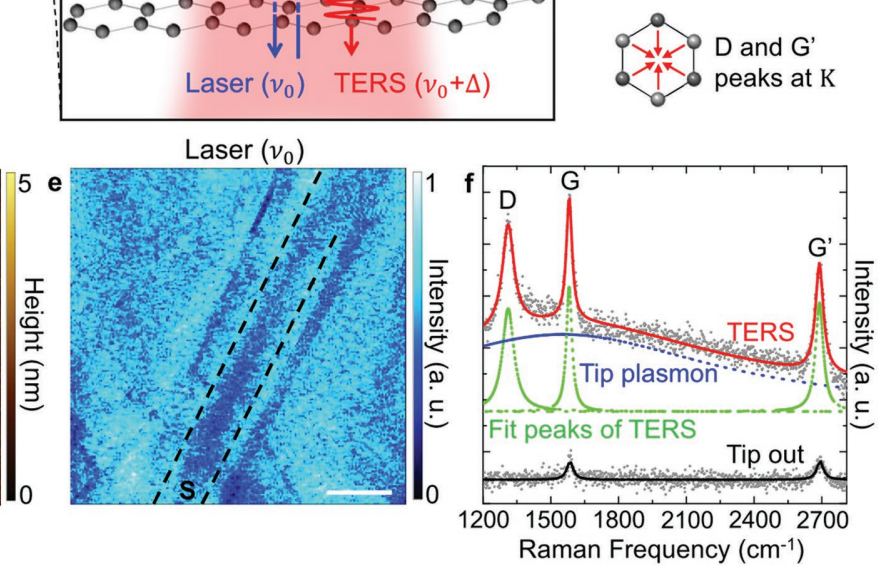
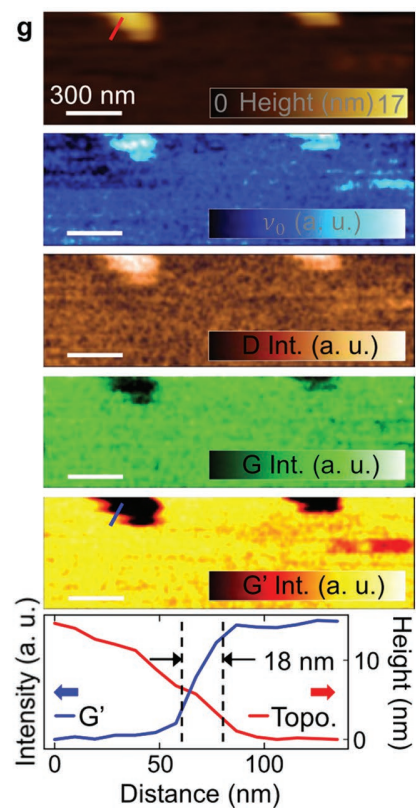

Figure 1. a) Schematic of multispectral TERS imaging (BS: beam splitter, M: mirror). b) Illustration of plasmon-enhanced light-matter interactions at the tip-graphene junction. c) Illustration of in-plane phonon modes of graphene. d) Topography of a CVD grown large-area graphene measured by shear force AFM exhibiting wrinkle (W) and grain boundary (GB) structures. e) Simultaneously measured tip-enhanced Rayleigh scattering image. f) TERS (red line) and confocal Raman spectra (black line) with Lorentzian line fit analysis. Blue and green dashed lines are tip plasmon and Raman spectra, respectively. g) Simultaneously measured multispectral TERS images of local defects (contaminations) exhibiting $\approx 18 \mathrm{~nm}$ spatial resolution.

ML graphene were measured as shown in Figure 1d,e. The scattering intensity was decreased at both wrinkle and GB structures due to the stronger absorption compared to $\mathrm{ML}$ regions. An additional dark stripe (indicated by $\mathrm{S}$, between the black dashed lines) was observed next to the GB in the scattering signal, but did not appear in the AFM topography. This dark stripe was generally observed for other GBs as well in our experiment (see Figure S1 of the Supporting Information for measurement results of other GBs).

Figure 1f shows a Lorentzian line fit analysis of the TERS and confocal Raman spectra obtained for a single-crystal grain region. The blue and green dashed lines show the tip plasmon spectrum and fit peaks of the three vibrational modes, derived from the TERS spectrum. The $G$ peak intensity is weaker than the $G^{\prime}$ peak in the confocal Raman measurement, but it is more enhanced in TERS due to a resonance Raman effect by the tip plasmon. ${ }^{[14,15]}$ In addition, the Raman D peak in the TERS spectrum appears consistently, though with varying intensity. A large contribution to the $\mathrm{D}$ peak likely arises due to an induced artificial defect, from the highly localized electromagnetic field at the tip apex. ${ }^{[16]}$

To confirm the instrumental spatial resolution of the multispectral TERS imaging setup, we performed test measurements of local defects on graphene as shown in Figure 1g, as a similar experiment to a previous TERS study. ${ }^{[17]}$ A spatial resolution of $\approx 18 \mathrm{~nm}$ was achieved in the $\mathrm{G}^{\prime}$ peak intensity image, and the smooth change in height in the simultaneously measured topography confirmed that this optical spatial resolution was not resulted from topographic artifacts.

Figure $2 b-f$ show the near-field Rayleigh scattering image and TERS responses, which reveal structural properties of complex GB formations not readily resolved in the AFM topography
(Figure 2a). The distinguishable line structures from topography are indicated by white dashed lines in the Rayleigh scattering (Figure $2 b$ ) and TERS images (Figure 2c-f). As can be seen in Figure $2 \mathrm{~b}$, several GBs show $\mathrm{a} \approx 1 \mu \mathrm{m}$ scale modification of Rayleigh scattering on one side of them. In addition, TERS images of the $D, G$, and $G^{\prime}$ peaks show a distinct Raman response at various $\mathrm{GBs}$, labeled $\mathrm{A}, \mathrm{B}$, and $\mathrm{C}$. The TERS $\mathrm{G} / \mathrm{G}^{\prime}$ intensity ratio image (Figure $2 \mathrm{f}$ ) best exemplifies the heterogeneity of the GBs. For fast TERS mapping, a large pixel size (100 nm per pixel) is used, yet the observed length scales at GBs are not limited by spatial resolution. Figure $2 \mathrm{~g}$ shows TERS spectra and Lorentzian line fit analysis at A, B, C, and grain regions. In contrast to the TERS images, confocal Raman images measured as same experimental conditions do not give a clear fingerprint of GBs due to the weaker intensity and lower spatial resolution than TERS (see Figure S2 of the Supporting Information for confocal Raman images).

It should be noted that the line defects of folded grains are possibly produced in large-area CVD-grown graphene. ${ }^{[18]}$ However, the possibility of folded structures could be explicitly excluded for the line defects A, B, and C from the correlated analysis of topography, near-field scattering, and TERS properties (see the Supporting Information for explanation in detail). While we could not observe the folded structures in the measured area of Figure 2, we could find the folded line defect in other measured area as shown in Figure S4 in the Supporting Information.

Figure 3a-d shows detailed vibrational properties at GBs A, $\mathrm{B}$, and $\mathrm{C}$, compared to the $\mathrm{ML}$ grain region. The distinguishing features of Rayleigh scattering and Raman responses at GBs $\mathrm{A}, \mathrm{B}$, and $\mathrm{C}$ suggest a bilayer structure. In general, the Raman response of Bernal (AB) stacked bilayer graphene shows an asymmetric $G^{\prime}$ peak, with decreased intensity, and broader full 

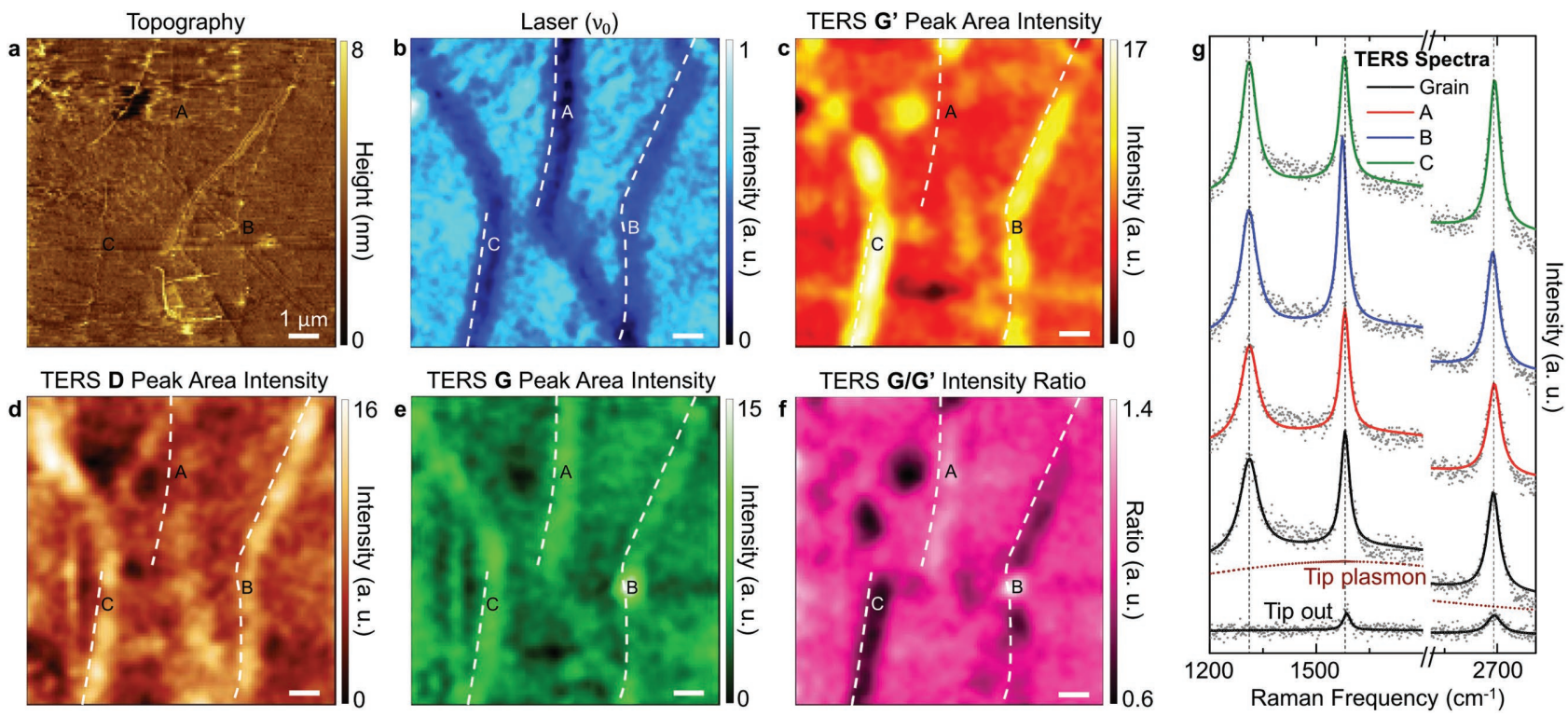

Figure 2. a) Topography of a CVD grown graphene exhibiting structural defects such as wrinkles and grain boundaries. b) Tip-enhanced Rayleigh scattering image. Distinct grain boundary structures of the topography (a) are marked with white dashed lines. $G^{\prime}$ peak area intensity (c), D peak area intensity (d), G peak area intensity (e), and TERS images of $\mathrm{G} / \mathrm{G}^{\prime}$ intensity ratio (f) are derived from multispectral TERS imaging (0.5 $\mathrm{s}$ acquisition time per pixel), measured simultaneously with (a) and (b). g) Lorentzian line fit analysis of the TERS and confocal Raman spectra for grain and GBs $\mathrm{A}, \mathrm{B}$, and $\mathrm{C}$ with an acquisition time of $5 \mathrm{~s}$.

width at half maximum (FWHM).[19] However, the observed $\mathrm{G}^{\prime}$ peaks at GBs exhibit neither weaker intensity nor distinctly broader FWHM compared to the $\mathrm{G}^{\prime}$ peak inside a grain. Therefore, the measured GBs A, B, and C are possibly formed as a twisted bilayer with varying misorientation angle $\theta$. As seen in Figure $3 a, b$, the $G^{\prime}$ peak intensity and FWHM are anticorrelated for A-C. In contrast, the $\mathrm{G}^{\prime}$ peak position at $\mathrm{B}$ is distinctly lower than A and C, as shown in Figure 3d.

From the quantitative analysis of relative vibrational properties, the stacking angles of bilayer GBs A, B, and C can be determined. The alternation of the $\mathrm{G}^{\prime}$ peak intensity in bilayer graphene with respect to the stacking angle was demonstrated experimentally ${ }^{[21,22]}$ and theoretically ${ }^{[20]}$ in previous studies. Since the magnitude of band-structure modification in bilayer graphene is dependent on the overlapping ratio of the Dirac cones of the top and bottom layers, the $G^{\prime}$ peak intensity of bilayer graphene gradually increases with the misorientation angle $\theta$ (from $0^{\circ}$ to $30^{\circ}$ ). ${ }^{[21]}$ The FWHM of the $\mathrm{G}^{\prime}$ peak is generally inversely proportional to the peak intensity except for the narrow zone near critical angle $\theta_{c}$, where the excitation photon energy $\left(E_{\text {laser }}\right)$ is the same as the energy between the conduction and valence Van Hove singularities. ${ }^{[21,22]}$ The $G^{\prime}$ peak position is
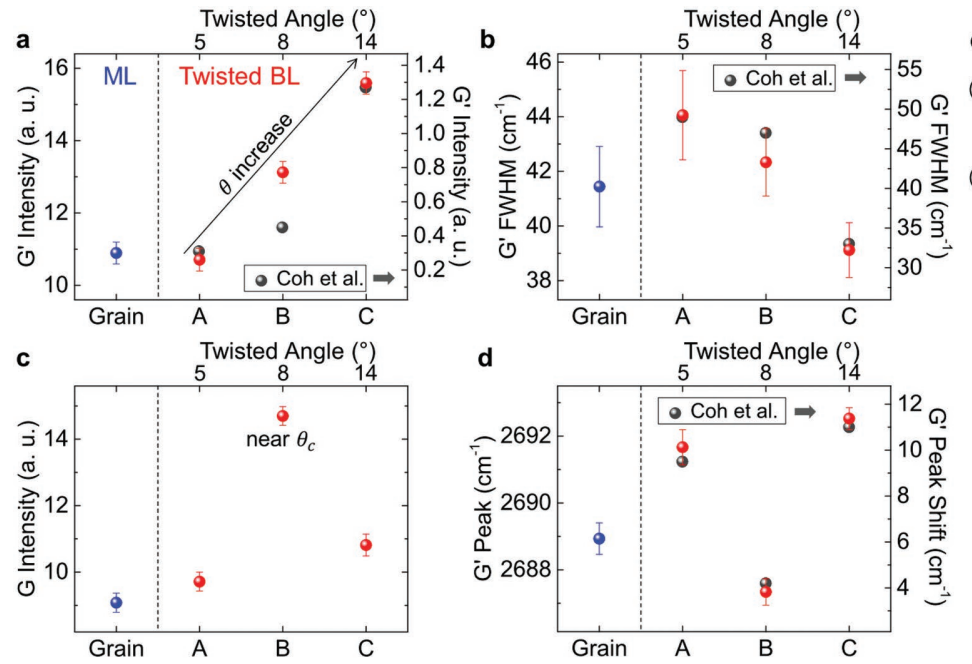
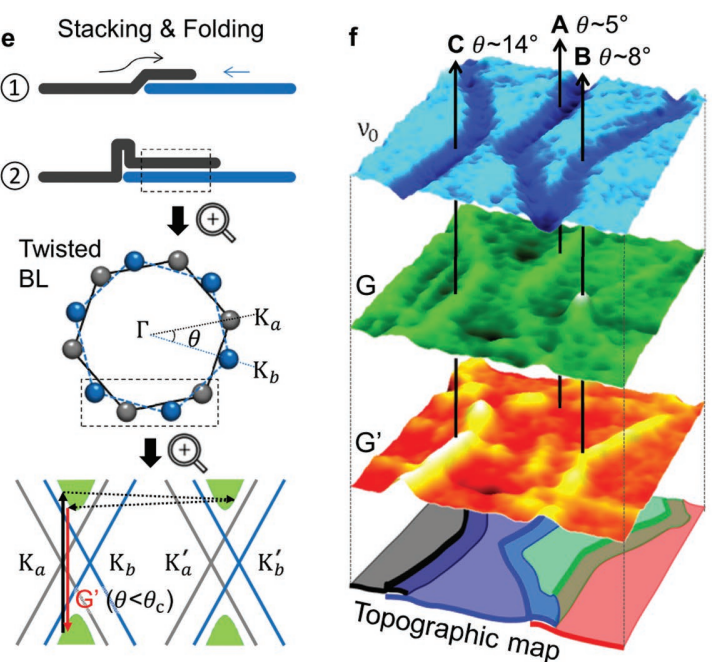

Figure 3. a-d) Quantitative analysis of vibrational properties for grain and $G B$ s $A, B$, and $C$ (all values are derived from Lorentzian line fitting of Figure $2 \mathrm{~g}$ ). Measured values of $\mathrm{G}^{\prime}$ peak at $\mathrm{GBs}$ (red circles) are compared with the calculated ones (gray circles) from Coh et al..[20] e) Illustration of formation and $\mathrm{G}^{\prime}$ peak Raman process at $\mathrm{GBs}$ in CVD grown large-area graphene. $f$ ) Determined misorientation angles of $G B s \mathrm{~A}, \mathrm{~B}$, and $\mathrm{C}$. 
also strongly influenced by the resonance near $\theta_{c}$, based on the general tendency of blueshift at small misorientation angles $\left(<7^{\circ}\right) \cdot{ }^{[20]}$ From comparison between the measured vibrational properties of the $\mathrm{G}^{\prime}$ peak and the calculated data by the super-cell tight-binding method, ${ }^{[20]}$ the misorientation angles of the observed GBs are determined as shown in Figure 3a,b,d. In the case of the boundary A, the $G^{\prime}$ peak is slightly weaker in intensity and larger in FWHM compared to ML region as results from the significantly modified band structure. In contrast, the $\mathrm{G}^{\prime}$ peak intensity is significantly increased at boundary $\mathrm{C}$ with a decreased FWHM due to the relatively weak interlayer coupling. From the best matching of the $G^{\prime}$ peak intensity and FWHM with the calculated data, we determine the misorientation angle of boundary A $\left(5^{\circ}\right), \mathrm{B}\left(8^{\circ}\right)$, and C $\left(14^{\circ}\right)$. Despite us not considering the $\mathrm{G}^{\prime}$ peak position in the angle determination, the spectral shift tendency of boundary A, B, and $\mathrm{C}$ is in good agreement with the calculated data. For $1.96 \mathrm{eV}$ excitation, a critical angle of $\approx 10^{\circ}$ is calculated by a Dirac dispersion relation given by: ${ }^{[21]}$

$\theta_{\mathrm{c}}=\frac{\Delta k}{K}=\frac{3 a E_{\text {laser }}}{\hbar v_{\mathrm{f}} 4 \pi}$

where $a$ and $v_{\mathrm{f}}$ are the lattice parameter $(2.46 \AA)$ and the Fermi velocity $\left(10^{6} \mathrm{~m} \mathrm{~s}^{-1}\right)$ of monolayer graphene, and $\hbar$ is the reduced Planck's constant. When the misorientation angle of bilayer graphene is close to the $\theta_{c}$, a drastic increase in $G$ peak intensity has been observed due to the resonance excitation of electron-hole, whereas the FWHM and position are not significantly changed with respect to the misorientation angle. ${ }^{[20,21,23]}$ This suggests that the observed strong $G$ peak intensity at $B$ (Figure 2e and Figure 3c) is possibly resulted from the resonance Raman effect near $\theta_{c}$.

In order to demonstrate the complete picture of the measured region, we also analyze several other line defects other than GBs A, B, and C such as the line between B and C, and the line to the left of the A as shown in Figure S5 (see Supporting Information for detailed analysis).

Furthermore, the other type of line defects in the topography can be resolved, suggesting wrinkle structures in the graphene sheets. We perform multispectral TERS imaging with $6 \mathrm{~nm}$ pixel size to investigate the Raman properties of the wrinkles. Figure $4 \mathrm{a}$,b shows topography and $\mathrm{G}^{\prime}$ peak intensity image of a wrinkle in the graphene (D and $G$ peaks' intensity images are shown in Figure S6 in the Supporting Information). As can be seen from comparing the TERS spectra (Figure 4c) of the wrinkle and grain regions, and their Lorentzian line fit analysis (Figure $4 d$ ), only the $\mathrm{G}^{\prime}$ peak exhibits significant changes in the vicinity of the wrinkle, with an increase in FWHM and a decrease in intensity, with no associated spectral shift. Note that the measured wrinkle is created in the CVD growth process due www.advancedsciencenews.com
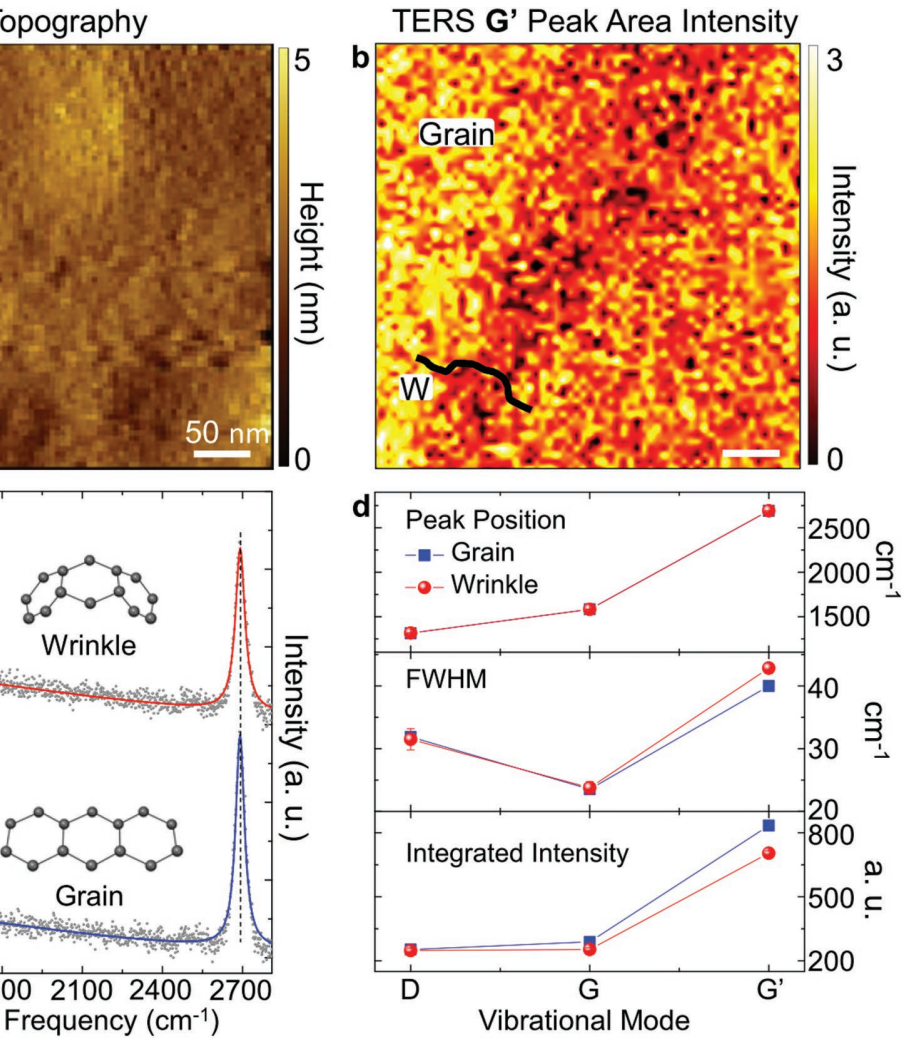

Vibrational Mode substrate and graphene ${ }^{[1,3,13,24]}$ (see the Supporting Information for details).

NSs in large-area graphene are also important defects that can provide valuable information regarding the growth mechanism. ${ }^{[3,13]}$ Figure 5a-f shows the AFM topography, near-field Rayleigh scattering image, and TERS images of a region in the vicinity of an NS. From the modification of near-field scattering properties (Figure 5b), the NS is interpreted as a multilayer graphene region. Specifically, the sub-micrometer region of decreased scattering intensity at the center of the grain is thought to be the origin of nucleation. The strong $\mathrm{G}$ peak intensity in the center of the graphene grain (Figure 5c) supports this interpretation. On the other hand, the $\mathrm{G}^{\prime}$ peak intensity is decreased in the vicinity of the NS (Figure 5e). Figure $5 \mathrm{~g}$ shows details of the vibrational properties of the NS region through the spectral line trace (indicated by yellow line in Figure 5e). At the multilayer region (indicated by $F$ ), the $\mathrm{G}^{\prime}$ peak has decreased intensity and increased FWHM, and the peak position is blueshifted in comparison with the ML region (D), with asymmetric spectral shape. The integrated intensity of the $G$ peak is increased, with a slight redshift. These observed Raman properties are in good agreement with those characteristic of $A B$ stacking in bilayer graphene. ${ }^{[19]}$ In addition, the $\mathrm{G}^{\prime}$ peak intensity is decreased in the region labeled $\mathrm{E}$, with the emergence of an additional Raman peak at $\approx 1615 \mathrm{~cm}^{-1}$ (Figure $5 f$ ). This is the 

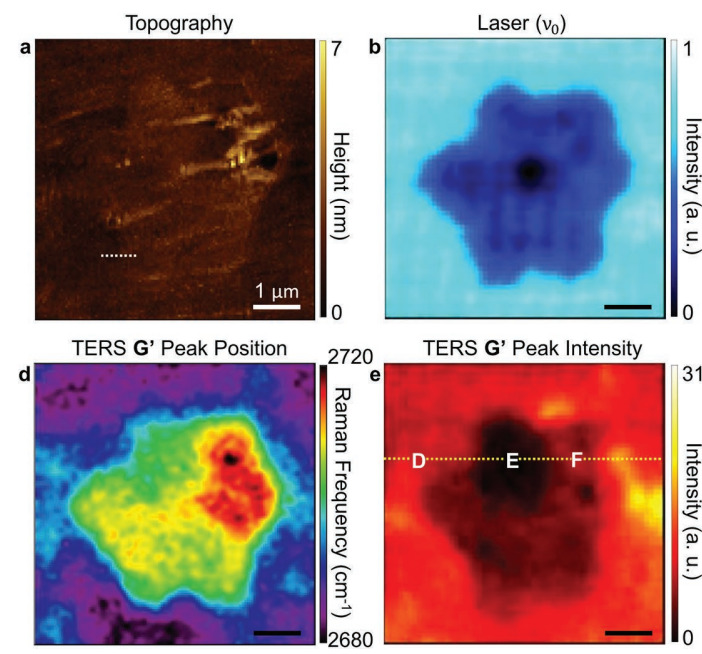

TERS G' Peak Intensity

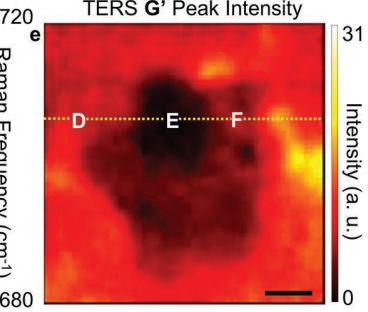

TERS G Peak Area Intensity

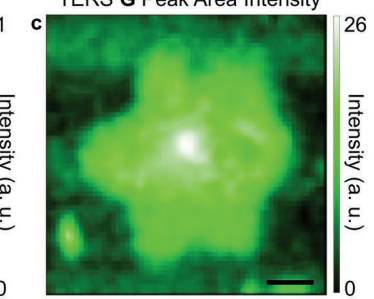

TERS D' Peak Area Intensity

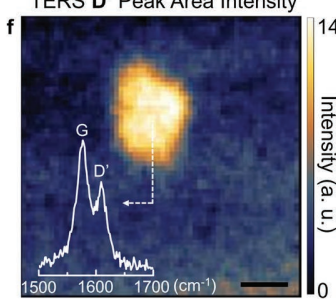

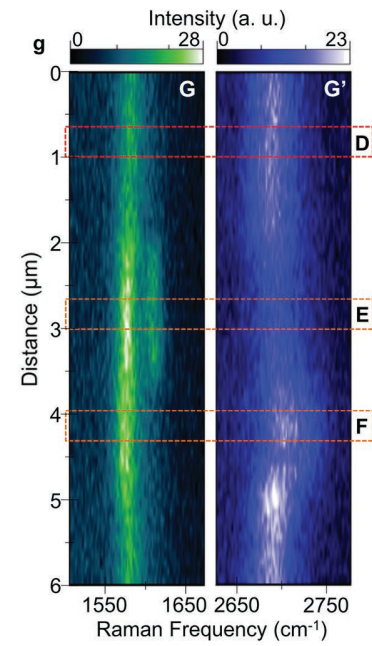

Figure 5. a) Topography of nucleation seed. b) Tip-enhanced Rayleigh scattering image simultaneously measured with (a). TERS images of $G$ peak area intensity (c), $\mathrm{C}^{\prime}$ peak position (d), $\mathrm{G}^{\prime}$ peak intensity (e), and $\mathrm{D}^{\prime}$ peak area intensity (f) are derived from the multispectral TERS imaging (0.5 $\mathrm{s}$ acquisition time per pixel). g) TERS spectral line traces of $G$ and $G^{\prime}$ peaks (yellow dotted line of (e)) with the cartoons of inferred structures at local spots D, E, and F.

$\mathrm{D}^{\prime}$ peak, generally described as a feature of hydrogen-bonded $\mathrm{sp}^{3}$ type defects, which depend on the growth conditions. ${ }^{[25,26]}$

In the following, we discuss the detailed analysis of the GBs, wrinkles, and NSs separately. From these studies, we determine that GB structures in large-area CVD-grown graphene are not similar to the well-known atomic vacancy defects observed at the boundaries of adjacent graphene flakes. Despite some STM studies showing evidence of bilayer GBs in large-area graphene, the structure and formation mechanism had not yet been determined. ${ }^{[9,10]}$ From correlated analysis of topography, near-field scattering, and multispectral TERS images, we confirm the twisted bilayer structure of GBs, as seen in Figure 3, and can assign misorientation angles for the overlapping regions. The misorientation angles of bilayer GBs are in the range from $0^{\circ}$ to $30^{\circ}$, depending on the orientation of the crystal faces. Since the Raman $\mathrm{G}^{\prime}$ peak originates from an intervalley phonon scattering between the $\mathrm{K}$ and $\mathrm{K}^{\prime}$ points, its response is sensitively changed with respect to the modified band structure. Thus, we could use the modified Raman properties of the $G^{\prime}$ peak to determine the misorientation angle as seen in Figure 3a,b,d.

The supercell tight-binding method of determining the misorientation angle provided a better fit to our data than the continuum method. ${ }^{[20]}$ The misorientation angles of bilayer graphene could be obtained most precisely for angles smaller than $6^{\circ}$ or larger than $15^{\circ}$, but there are large uncertainties for $\theta$ larger than $6^{\circ}$ and smaller than $15^{\circ}$ due to the non-monotonic change of peak position and FWHM near the critical angle $\theta_{\mathrm{c}}$. Despite the fact that the physical origin for the modified $\mathrm{G}^{\prime}$ peak responses at the misorientation angles smaller than $\theta_{c}$ is still not revealed, the possible scattering path between the overlapping Dirac cones was expected as described in Figure 3e. ${ }^{[21]}$ Meanwhile, for misorientation angles larger than $15^{\circ}$, the Raman responses would be expected to resemble the monolayer properties since the Raman process occurs in isolated Dirac cones without overlapping. ${ }^{[21,22]}$

Based on the variation we observe, we infer a formation mechanism for GBs in large-area graphene. As described in Figure $3 \mathrm{e}$, one of two facing grains ascends onto the other grain, and makes a twisted bilayer region on the length scale of several hundreds of nanometers. The top and bottom grains stop growing further due to friction, and the part of the top grain above the edge of the bottom grain is pushed up by a surface horizontal compressive force. It should be noted that since the vertical resolution of a shear-force AFM is $\approx 1 \mathrm{~nm}$, we quantify the height of $\approx 2 \mathrm{~nm}$ at the pushed-up structure of GBs, whereas we cannot clearly quantify the thickness difference of $0.35 \mathrm{~nm}$ between monolayer grains and the GBs at the other edges.

Wrinkles are also significant nanoscale defects that degrade the electrical properties of large-area graphene. While the structural and electrical properties of wrinkles have been studied by $\mathrm{STM}^{[27]}$ the vibrational properties of single wrinkle structures had not previously been investigated. As seen in Figure 4, there are no obvious spectral changes in the $\mathrm{D}$ and $\mathrm{G}$ peaks at a wrinkle structure, with only the $\mathrm{G}^{\prime}$ peak decreasing in intensity and increasing in linewidth. These modified vibrational properties of the $G^{\prime}$ peak could be interpreted as a simple curvature effect similar to the suppressed $\mathrm{G}^{\prime}$ peak intensity of carbon nanotubes $(\mathrm{CNT}){ }^{[28]}$ In addition, the unchanged $\mathrm{G}^{\prime}$ peak position means that the wrinkle structures have monolayer thickness. ${ }^{[28]}$

In the nucleation process of CVD graphene, $\mathrm{sp}^{3}$ type defects associated with the $\mathrm{H}_{2}$ precursor are expected to be formed. ${ }^{[25,26]}$ Recently, $\mathrm{sp}^{3}$ defects were experimentally observed by $\mathrm{STM}^{[29]}$ and Raman spectroscopy, ${ }^{[30]}$ for artificially treated graphene samples. From the D' peak, our results show the first TERS imaging of hydrogen-bonded $\mathrm{sp}^{3}$ defects at the NS of unmodified graphene (Figure 5f). Note that while the $\mathrm{D}^{\prime}$ peak also emerges from vacancy-type defects, the intensity ratio of the $\mathrm{D}^{\prime}$ and $\mathrm{G}$ peaks is relatively small in those cases. ${ }^{[30]}$ In addition, since the intensity ratio of $\mathrm{D}^{\prime} / \mathrm{G}$ (inset of Figure $5 \mathrm{f}$ ) is comparable to a previous study, ${ }^{[30]}$ we can conclude that the observed $\mathrm{D}^{\prime}$ at the NSs is a fingerprint of the $\mathrm{sp}^{3}$-type defects. Additionally, the spectral redshift of the $\mathrm{G}^{\prime}$ peak is also supporting evidence of the $\mathrm{sp}^{3}$ type defects, as seen in Figure 5d. ${ }^{[30]}$ Although a complete physical origin of the observed heterogeneous distribution could not be addressed, we expect this observation gives clues to understand the initial growth mechanism of graphene and graphane. 
Our proposed method shows the potential of correlated TERS analysis as generally applicable to a wide range of 2D layered materials and heterostructures beyond graphene. For example, structures and electronic/vibrational properties at GBs and NS of recently synthesized large-area transition metal dichalcogenides $(\text { TMDs })^{[31]}$ could be revealed through correlated TERS analysis.

In summary, we have revealed twisted bilayer structures of grain boundaries in large-area graphene and identified misorientation angles from correlated analysis of the highresolution $(\approx 18 \mathrm{~nm})$ multispectral TERS imaging. In addition, we have investigated phonon-scattering properties of wrinkles and nucleation sites, which are associated with the nanoscopic structural curvature effect and atomic-scale carbon hybridization. We expect these results help to better understand nanoscale defects, their impact on function, and growth mechanisms of large-area graphene.

\section{Experimental Section}

Sample Preparation: Large-area graphene was grown on a $100 \mu \mathrm{m}$ thick copper foil using CVD. The chamber was heated up to $1100{ }^{\circ} \mathrm{C}$ in $1000 \mathrm{sccm}$ of $\mathrm{Ar}$ and $200 \mathrm{sccm}$ of $\mathrm{H}_{2}$ environment, then annealing was maintained for $20 \mathrm{~min}$. $\mathrm{CH}_{4}$ gas was then injected at a reduced pressure of $\mathrm{H}_{2}$ to nucleate and grow graphene. The sample was cooled down to room temperature with an optimized cooling rate. The as-grown sample was then transferred onto a glass cover slip. Details can be found in ref. [13].

TERS Setup: Figure 1a,b shows a schematic of TERS setup. The sample is mounted to a piezoelectric transducer (PZT) for xyz scanning, with a digital controller (NTEGRA spectra, NT-MDT). Au tips were electrochemically etched with $\approx 10 \mathrm{~nm}$ apex radius, and glued to a quartz tuning fork (resonance frequency $\approx 32 \mathrm{kHz}$ ). ${ }^{[32]}$ For shear-force feedback, the tuning fork/Au tip assembly was mechanically driven at its resonance frequency and the amplitude was monitored for tip-sample distance control. The tip-enhanced Raman signal was collected in backscattered direction, passed through an edge filter (633 nm cut-off) and detected using a spectrometer ( $f=300 \mathrm{~mm}$, NT-MDT, 600 grooves $\mathrm{mm}^{-1}$ grating) with a thermoelectrically cooled charge-coupled device (Andor). The nearfield Rayleigh scattering signal was detected by a photomultiplier tube.

\section{Supporting Information}

Supporting Information is available from the Wiley Online Library or from the author.

\section{Acknowledgements}

The authors thank jin Ho Park for help with Au tips fabrication. The authors also thank Ki Kang Kim and Doo Jae Park for valuable discussions. This work was in part supported by the IBS-R011-DI of Korea. K.-D.P and M.B.R. acknowledge support from the U.S. Department of Energy, Office of Basic Sciences, Division of Material Sciences and Engineering, under Award No. DE-SC0008807. The authors declare no competing financial interest.

Received: July 8, 2016 Revised: October 4, 2016 Published online:

[1] X. Li, W. Cai, J. An, S. Kim, J. Nah, D. Yang, R. Piner, A. Velamakanni, I. Jung, E. Tutuc, S. K. Banerjee, L. Colombo, R. S. Ruoff, Science 2009, 324, 1312 .

[2] F. Bonaccorso, Z. Sun, T. Hasan, A. Ferrari, Nat. Photonics 2010, 4, 611.
[3] S. J. Chae, F. Günes, K. K. Kim, E. S. Kim, G. H. Han, S. M. Kim, H.-J. Shin, S.-M. Yoon, J.-Y. Choi, M. H. Park, C. W. Yang, D. Pribat, Y. H. Lee, Adv. Mater. 2009, 21, 2328.

[4] K. Kim, Z. Lee, W. Regan, C. Kisielowski, M. Crommie, A. Zettl, ACS Nano 2011, 5, 2142.

[5] D. L. Duong, G. H. Han, S. M. Lee, F. Gunes, E. S. Kim, S. T. Kim, H. Kim, Q. H. Ta, K. P. So, S. J. Yoon, S. J. Chae, Y. W. Jo, M. H. Park, S. H. Chae, S. C. Lim, J. Y. Choi, Y. H. Lee, Nature 2012, 490, 235.

[6] A. W. Tsen, L. Brown, M. P. Levendorf, F. Ghahari, P. Y. Huang, R. W. Havener, C. S. Ruiz-Vargas, D. A. Muller, P. Kim, J. Park, Science 2012, 336, 1143.

[7] O. V. Yazyev, S. G. Louie, Nat. Mater. 2010, 9, 806.

[8] Q. Yu, L. A. Jauregui, W. Wu, R. Colby, J. Tian, Z. Su, H. Cao, Z. Liu, D. Pandey, D. Wei, T. F. Chung, P. Peng, N. P. Guisinger, E. A. Stach, J. Bao, S.-S. Pei, Y. P. Chen, Nat. Mater. 2011, 10, 443.

[9] W. Yan, W.-Y. He, Z.-D. Chu, M. Liu, L. Meng, R.-F. Dou, Y. Zhang, Z. Liu, J.-C. Nie, L. He, Nat. Commun. 2013, 4, 2159.

[10] J. C. Koepke, J. D. Wood, D. Estrada, Z.-Y. Ong, K. T. He, E. Pop, J. W. Lyding, ACS Nano 2013, 7, 75.

[11] P. Y. Huang, C. S. Ruiz-Vargas, A. M. van der Zande, W. S. Whitney, M. P. Levendorf, J. W. Kevek, S. Garg, J. S. Alden, C. J. Hustedt, Y. Zhu, J. Park, M. P. L., D. A. Muller, Nature 2011, 469, 389.

[12] I. N. Kholmanov, C. W. Magnuson, A. E. Aliev, H. Li, B. Zhang, J. W. Suk, L. L. Zhang, E. Peng, S. H. Mousavi, A. B. Khanikaev, R. Piner, G. Shvets, R. S. Ruoff, Nano Lett. 2012, 12, 5679.

[13] G. H. Han, F. Gunes, J. J. Bae, E. S. Kim, S. J. Chae, H.-J. Shin, J.-Y. Choi, D. Pribat, Y. H. Lee, Nano Lett. 2011, 11, 4144.

[14] K.-D. Park, E. A. Muller, V. Kravtsov, P. M. Sass, J. Dreyer, J. M. Atkin, M. B. Raschke, Nano Lett. 2016, 16, 479.

[15] K.-D. Park, O. Khatib, V. Kravtsov, G. Clark, X. Xu, M. B. Raschke, Nano Lett. 2016, 16, 2621.

[16] P. Wang, D. Zhang, L. Li, Z. Li, L. Zhang, Y. Fang, Plasmonics 2012, 7, 555 .

[17] J. Stadler, T. Schmid, R. Zenobi, ACS Nano 2011, 5, 8442.

[18] W. Zhu, T. Low, V. Perebeinos, A. A. Bol, Y. Zhu, H. Yan, J. Tersoff, P. Avouris, Nano Lett. 2012, 12, 3431.

[19] K. M. Subhedar, I. Sharma, S. R. Dhakate, Phys. Chem. Chem. Phys. 2015, 17, 22304

[20] S. Coh, L. Z. Tan, S. G. Louie, M. L. Cohen, Phys. Rev. B 2013, 88, 165431.

[21] K. Kim, S. Coh, L. Z. Tan, W. Regan, J. M. Yuk, E. Chatterjee, M. Crommie, M. L. Cohen, S. G. Louie, A. Zettl, Phys. Rev. Lett. 2012, 108, 246103.

[22] Y. Chen, L. Meng, W. Zhao, Z. Liang, X. Wu, H. Nan, Z. Wu, S. Huang, L. Sun, J. Wang, Z. Ni, Phys. Chem. Chem. Phys. 2014, 16, 21682.

[23] R. W. Havener, H. Zhuang, L. Brown, R. G. Hennig, J. Park, Nano Lett. 2012, 12, 3162.

[24] N. Liu, Z. Pan, L. Fu, C. Zhang, B. Dai, Z. Liu, Nano Res. 2011, 4, 996.

[25] L. Tsetseris, S. T. Pantelides, J. Mater. Sci. 2012, 47, 7571.

[26] M. Losurdo, M. M. Giangregorio, P. Capezzuto, G. Bruno, Phys. Chem. Chem. Phys. 2011, 13, 20836.

[27] K. Xu, P. Cao, J. R. Heath, Nano Lett. 2009, 9, 4446.

[28] A. Ferrari, J. Meyer, V. Scardaci, C. Casiraghi, M. Lazzeri, F. Mauri, S. Piscanec, D. Jiang, K. Novoselov, S. Roth, A. Geim, Phys. Rev. Lett. 2006, 97, 187401.

[29] C. Lin, Y. Feng, Y. Xiao, M. Durr, X. Huang, X. Xu, R. Zhao, E. Wang, X.-Z. Li, Z. Hu, Nano Lett. 2015, 15, 903.

[30] A. Eckmann, A. Felten, A. Mishchenko, L. Britnell, R. Krupke, K. S. Novoselov, C. Casiraghi, Nano Lett. 2012, 12, 3925.

[31] K. Kang, S. Xie, L. Huang, Y. Han, P. Y. Huang, K. F. Mak, C.-J. Kim, D. Muller, J. Park, Nature 2015, 520, 656.

[32] C. C. Neacsu, J. Dreyer, N. Behr, M. B. Raschke, Phys. Rev. B 2006, $73,193406$. 